\title{
Uso da Técnica de Ressonância de Barras no Monitoramento das Propriedades Mecânicas da Zona Fundida do Aço ASTM A36
}

Bruno Duarte ${ }^{1}$ (D), Waldemir dos Passos Martins ${ }^{1}$ (D), Amadeu Nunes Santos ${ }^{1}$, Catarine Tayane Caetano Santos da Silva ${ }^{1}$, Valdemar Silva Leal $^{1}$

${ }^{1}$ Instituto Federal de Educação, Ciência e Tecnologia do Maranhão - IFMA, São Luís, MA, Brasil.

Como citar: Duarte B, Martins WP, Santos AN, Silva CTCS, Leal VS. Uso da técnica de ressonância de barras no monitoramento das propriedades mecânicas da zona fundida do aço ASTM A36. Soldagem \& Inspeção. 2020;25:e2521. https://doi.org/10.1590/0104$9224 / \mathrm{SI} 25.21$

\begin{abstract}
Resumo: Neste estudo, faz-se uso do método dinâmico e a técnica de ressonância de barras para medir as constantes elásticas da ZF da junta soldada de um aço estrutural ASTM Grau A36. A geometria, massa e frequência de ressonância (na flexão e na torção), de amostras com seção transversal retangular, são usadas para determinar as características elásticas e homogeneidade da junta soldada e do metal base pela norma ASTM E1875-13. As demais propriedades da junta soldada são determinadas pelos ensaios convencionais de tração, impacto (Charpy V), dureza e por microscopia ótica, difração de raios-X e EDS acoplada ao MEV. Os resultados dos ensaios convencionais quantificaram valores dentro dos padrões estabelecidos por norma, demonstraram a formação de fase típica sem a formação de novas fases e a dureza média foi $10 \%$ maior na ZF, o comportamento de fratura se mostrou dúctil. O refino de grão na região revelou uma maior quantidade de perlita e maior teor de elementos de liga, as constantes elásticas foram alteradas em relação ao $\mathrm{MB}$ de 4,9\% a mais para o módulo de Young, 7,65\% maior para o cisalhamento e 11,7\% menor para a razão de Poisson, mostrando assim que o método é viável tanto para prever no MB e na ZF os valores das constantes elásticas.
\end{abstract}

Palavras-chave: Ressonância de barras; Ensaios não destrutivo; Razão de Poisson; Módulo de cisalhamento; Módulo de Young.

\section{Use of the Bar Resonance Technique in Monitoring the Mechanical Properties of the Melted Zone of ASTM A36 Steel}

\begin{abstract}
In this study, the bar resonance technique is used to measure the elastic constants of the welded joint of an ASTM Grade A36 structural steel. The geometry, mass and resonant frequency (flexion and torsion) of samples with rectangular cross section are used to determine the elastic characteristics and homogeneity of the welding and base metal by ASTM E1875-13.The other properties of the weld joint are determined by the conventional tests of traction, impact (Charpy V), hardness and microstructural characterization by optical microscopy, X-ray diffraction and EDS coupled to MEV. The results of the conventional tests quantified values within the norms established by the standard, demonstrated the typical phase formation without the formation of new phases and the average hardness was $10 \%$ higher in the ZF, the fracture behavior was ductile. The grain refining in the region revealed a higher amount of perlite and higher alloying content. The elastic constants were changed in relation to the $4.9 \%$ higher MB for Young's modulus, $7.65 \%$ higher for shear and $11.7 \%$ lower for Poisson's ratio, thus showing that the method is feasible to predict both MB and ZF elastic constant values
\end{abstract}

Key-words: Bar resonance; Non-destructive test; Poisson's ratio; Shear modulus; Young's modulus.

\section{Introdução}

Dentre os métodos utilizados para determinar as propriedades mecânicas dos materiais, pode-se ressaltar os métodos mecânicos de tensão e deformação convencionais, porém, o método dinâmico se mostra atrativo, pois apresenta menores incertezas, e é classificado como ensaio não destrutivo (END), cuja investigação se dá na frequência natural do material. Para a caracterização elástica sob ressonância, destaca-se a norma ASTM E1875-13 [1] que é uma norma completa e normaliza a caracterização elástica dos materiais elásticos, homogêneos e isotrópicos sob ressonância a temperatura ambiente e a alta temperatura. Amostras destes materiais possuem frequências mecânicas ressonantes específicas que são correlacionadas aos módulos de elasticidade, massa e geometria do corpo de prova. Desta forma, as propriedades elásticas dinâmicas de um

Recebido: 10 Fev., 2020. Aceito: 10 Jun., 2020.

E-mails: brunoduarte12@hotmail.com (BD), waldemir@ifma.edu.br (WPM)

Este é um artigo publicado em acesso aberto (Open Access) sob a licença Creative Commons Attribution Non-Commercial, que permite uso, distribuição e reprodução em qualquer meio, sem restrições desde que sem fins comerciais e que o trabalho original seja corretamente citado. 
material podem ser calculadas se as frequências (na flexão e na torção), geometria e massa da amostra do material puderem ser medidas.

O módulo de Young dinâmico é determinado usando a frequência de ressonância no modo de vibração de flexão. O módulo de cisalhamento dinâmico, ou módulo de rigidez, é encontrado usando vibrações ressonantes de torção. O módulo de Young e de cisalhamento são usados para calcular o coeficiente de Poisson. Entretanto, a própria norma já alerta para o fato de que este método não é satisfatório para amostras que apresentam trincas ou vazios, que são as maiores descontinuidades que normalmente se apresentam nos materiais soldados. Também não é satisfatório para amostras com seção transversal diferente do retangular ou circular. Porém, a modificação deste método de teste para uso no controle de qualidade é possível.

Em termos de controle de qualidade, uma faixa de frequência ressonante é aceitável quando determinada para uma amostra com uma geometria e massa específicas. Qualquer amostra com uma resposta de frequência que esteja fora dessa faixa de frequência é rejeitada. O módulo efetivo de cada amostra não precisa ser determinado, desde que os limites da faixa de frequência selecionada sejam conhecidos por incluir a frequência de ressonância que a amostra deve possuir se sua geometria e massa estiverem dentro das tolerâncias especificadas.

Entre as pesquisas relacionadas com a caracterização elástica pelo uso das frequências naturais de vibração, pode-se citar o trabalho de Paiva [2] que desenvolveu um equipamento capaz de determinar o módulo de Young, módulo de cisalhamento e razão de Poisson, de forma totalmente automática a temperatura ambiente e a alta temperatura. Pereira et al. [3] incrementaram o equipamento desenvolvido por Paiva [2] para a caracterização dos módulos elásticos e conseguiram resultados com uma diferença de $\pm 3 \%$ com relação aos valores da literatura do módulo de Young, cisalhamento e Poisson.

Mesmo de conhecimento da literatura especializada na área de END, o uso da técnica de ressonância de barras ainda é pouco explorado na soldagem, o que demonstra que seu uso nesta área se encontra aberto a maiores pesquisas para determinação de sua viabilidade, considerando que heterogeneidades decorrentes de descontinuidades comuns em juntas soldadas, como porosidades e trincas, podem influenciar nos resultados. Para isto, testes preliminares são necessários para validar os resultados uma vez que essa região é formada por dois materiais distintos. Logo, de acordo com o exposto, mostra-se como a medida das constantes elásticas, por meio da técnica de ressonância de barras, é afetada quando se compara o resultado do metal base (MB) e metal de solda (ZF). Portanto, o objetivo do presente trabalho, é monitorar a dinâmica na região da junta soldada multipasse, em termos das suas propriedades elásticas, usando o processo MIG/MAG, correlacionando-as ás suas propriedades mecânicas e microestruturais.

\section{Desenvolvimento}

\subsection{Materiais}

Neste trabalho foi utilizado um aço estrutural ASTM grau A36 de dimensões $300 \mathrm{~mm} \times 200 \mathrm{~mm} \times 20,5 \mathrm{~mm}$, cuja composição química e demais propriedades mecânicas são mostradas na Tabela 1. A soldagem das chapas foi realizada em junta de topo com chanfro em $V$ e ângulo do chanfro de $90^{\circ}$ como mostra a Figura 1 . Na soldagem do corpo de prova foi utilizado como consumível um arame sólido cobreado, norma AWS A5.18, do tipo MIG ER70S-6, de 1,2 mm de diâmetro, protegidos com a mistura gasosa $\mathrm{Ar}+25 \% \mathrm{CO}_{2}$ cuja composição química e demais propriedades mecânicas são mostradas na Tabela 2.

Tabela 1. Composição química (\% em peso) e propriedades mecânicas do aço estrutural A36.

\begin{tabular}{ccccccc}
\hline Composição & C & Mn & Si & Cu & P & S \\
\% Peso & 0,125 & 0,867 & 0,206 & 0,007 & 0,021 & 0,013 \\
Propriedade Mecânica & LR (MPa) & LE (MPa) & Alongamento & E (GPa) & G(GPa) & $\boldsymbol{\mu}$ \\
& $400-500$ & $250(\mathrm{~min})$ & $20 \%$ & 207 & 75 & 0,30 \\
\hline
\end{tabular}

Sendo: LR = Resistência a tração; LE = Resistência ao escoamento; $\mu$ = Razão de Poisson. Fonte: Norma ASTM A36 [4]. 


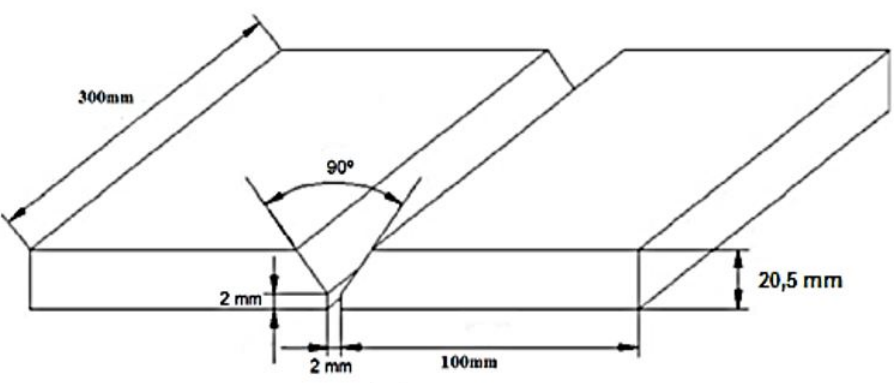

(a)

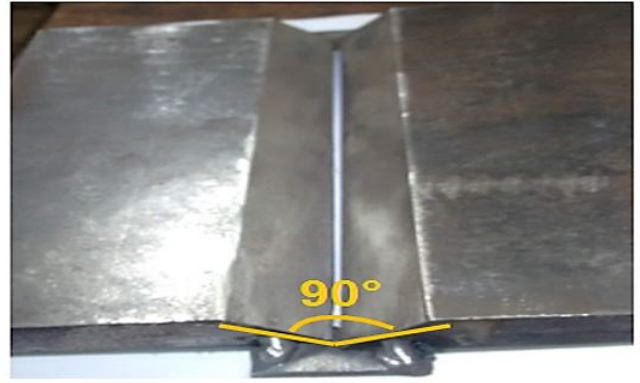

(b)

Figura 1. (a) Esboço esquemático do corpo de prova com as dimensões; (b) Chapa preparada para soldagem.

Tabela 2. Composição química (\% Peso) e propriedades mecânicas do arame ER70S-6.

\begin{tabular}{llcccccc}
\hline Composição & C & Mn & Si & Cu & S & Ni & Cr \\
\% peso & 0,09 & 1,6 & 0,9 & 0,20 & 0,0007 & 0,05 & 0,05 \\
Propriedades Mecânicas & & LR (MPa) & LE (MPa) & Alongamento & Tenacidade (20 $0^{\circ}$ ) \\
& 540 & 440 & $26 \%$ & 35J & \\
\hline
\end{tabular}

Sendo: $L R=$ Resistência a tração; LE = Resistência ao escoamento. Fonte: AWS A 5.18 [5].

\subsection{Métodos}

A soldagem foi realizada na posição plana, junta de topo, sentido puxando. Na Tabela 3, pode ser verificado os parâmetros de soldagem adotados neste trabalho:

Tabela 3. Valores dos parâmetros de soldagem usados no trabalho.

\begin{tabular}{|c|c|c|c|c|}
\hline $\mathrm{DBCP}(\mathrm{mm})$ & Vs (mm/min) & $\mathrm{Va}(\mathrm{mm} / \mathrm{min})$ & Ur (V) & Tipo de passe \\
\hline 18 & 210 & 3 & 20 & Raiz \\
\hline 16 & 290 & 3,5 & 28 & Enchimento \\
\hline
\end{tabular}

Simbologia: DBCP = Distância bico de contato-peça; Vs = Velocidade de soldagem; Va = Velocidade do arame; Ur = Tensão de referência.

Estes parâmetros garantem uma boa penetração de raiz bem como uma boa taxa de deposição. A preparação metalográfica dos corpos de prova para análise microscópica foi realizada de maneira convencional, sendo que na junta soldada foi realizada planificação da peça em retífica especializada com rebolo de granulométrica 800 mesh e com micrometro de divisão de $0,02 \mathrm{~mm}$. As amostras, depois de polidas e atacadas, foram analisadas microscopicamente por microscopia ótica com a finalidade de caracterizar e quantificar as fases presentes. A quantificação das fases foi realizada utilizando-se o software ImageJ.

A análise de DRX foi realizada no MB e na ZF. As amostras foram obtidas ao realizar um corte na região transversal da região da solda de maneira a definir a estrutura e as fases presentes nos planos cristalográficos de cada estado como recebido e como soldado.

O ensaio convencional de tração e impacto foi realizado no $\mathrm{MB}$ e na ZF e a preparação e execução dos ensaios em conformidade com as respectivas normas ASTM E384-17 [6] e ASTM E23-18 [7] e os ensaios realizados no Senai-MA. Após os ensaios de impacto e tração, as superfícies de fratura dos corpos de prova rompidos foram analisadas através de microscopia eletrônica de varredura (MEV) e (EDS) com o objetivo de monitorar o comportamento de fratura e a composição química dessas regiões.

Para a determinação do perfil de microdureza Vickers, com uma carga de 4.9N $(0,5 \mathrm{HV})(499,66 \mathrm{gf})$, foi utilizado um microdurômetro Shimizu HMV-2TE, as medidas de dureza foram realizadas nas mesmas amostras usadas na microscopia ótica e foram efetuadas em média 140 endentações em linha, tomando por base o centro da amostra, adotando-se como parâmetro os estudos de Tewary et al. [8] e Guzman-Flores et al. [9]. A distância entre uma endentação e outra adotada foi de $25 \mu \mathrm{m}$ ao longo de toda a amostra. Foram feitos perfis de microdureza (endentações) em linha vertical e horizontal na ZF e no MB totalizando 3 (três) linhas de endentações.

Para a caracterização elástica do MB e ZF, adotou-se a geometria de seção retangular e a posição de acoplamento são posicionados como mostra a Figura 2. Sobre a amostra, a uma distância de 0,224 x L de cada uma das suas extremidades, modo convencional e a varredura foram feitos sobre os apoios, visando diminuir o efeito de inércia de massa sobre a localização dos picos, fundamentais uma vez que a densidade desse tipo de material é maior que nos cerâmicos. 


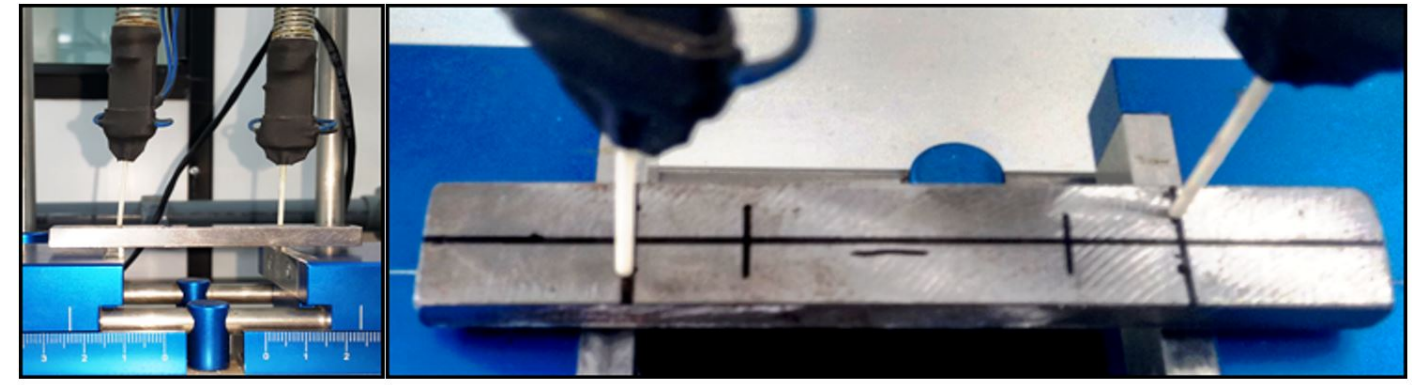

(a)

Figura 2. Acoplamento dos transdutores $0,224 \times \mathrm{L}$ (a) vista frontal; (b) vista superior.

Como o acoplamento foi feito de forma não convencional, foi empregada a metodologia adotada por Suterio [10], para o monitoramento da influência da distância do acoplamento no MB. Para verificar a influência da distância do acoplamento dos transdutores emissor e receptor foi quantificado a frequência de ressonância (modo flexional e torcional) e o coeficiente de Poisson em função da variação da distância entre os cutelos de apoio. Um conjunto de medidas foi realizado no MB. A Figura 3 mostra o esquema das medidas realizadas. A distância de $48,85 \mathrm{~mm}$ é a distância nominal para um corpo de prova com $88,5 \mathrm{~mm}$ de comprimento. Na Tabela 4 podem ser vistos os valores das geometrias do MB e ZF.

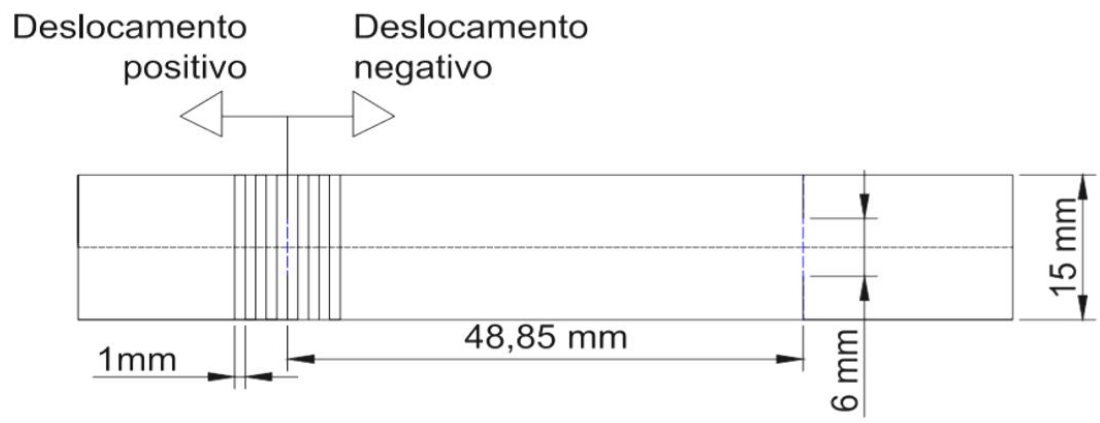

Figura 3. Esquema de marcação na quantificação das constantes elásticas.

Foi designada como deslocamento negativo a leitura que se aproximaram no sentido do centro da amostra e deslocamento positivo sentido inverso.

Tabela 4. Dimensões dos corpos de prova utilizados para ensaios no Scanelastic MB e na ZF.

\begin{tabular}{cccccc}
\hline Amostra & Comprimento (L) $\mathbf{~ m m}$ & Largura (b) $\mathbf{~ m m}$ & Espessura (t) $\mathbf{m m}$ & Massa (g) \\
MB & 88,5 & 15 & 6,5 & 65 \\
ZF & 88,5 & 15 & 6,5 & 67,427 \\
\hline
\end{tabular}

Sendo: $\mathrm{MB}=$ Metal base; $\mathrm{ZF}=$ Zona fundida.

Como descrito nos trabalhos de Chanbi et al. [11] e Bao et al. [12], foram realizados vários testes objetivando verificar os resultados de $\mathrm{E}(\mathrm{GPa}), \mathrm{G}(\mathrm{GPa})$ e Poisson, alinhando a localização dos picos com as razões entre picos esperado/real objetivando menor erro e menor desvio padrão entre cada leitura. Também, as amostras foram giradas em várias direções no plano, isso foi feito para verificar se os espectros de resposta à vibração eram os mesmos e assim verificar a homogeneidade/isotropia das amostras no MB e na região da ZF. Para verificar a influência de isotropia dos testes realizados, recorreu-se a análise estatística de regressão linear Likhachev [13], 5 (cinco) resultados de frequência (flexional e torcional) foram coletados em cada direção e quantificados em função dos valores de módulo de Young e cisalhamento.

\section{Resultados e Discussão}

Na Figura 4 é possível observar a sequência de deposição dos passes, primeiro o de raiz seguindo com deposição e acabamento. 


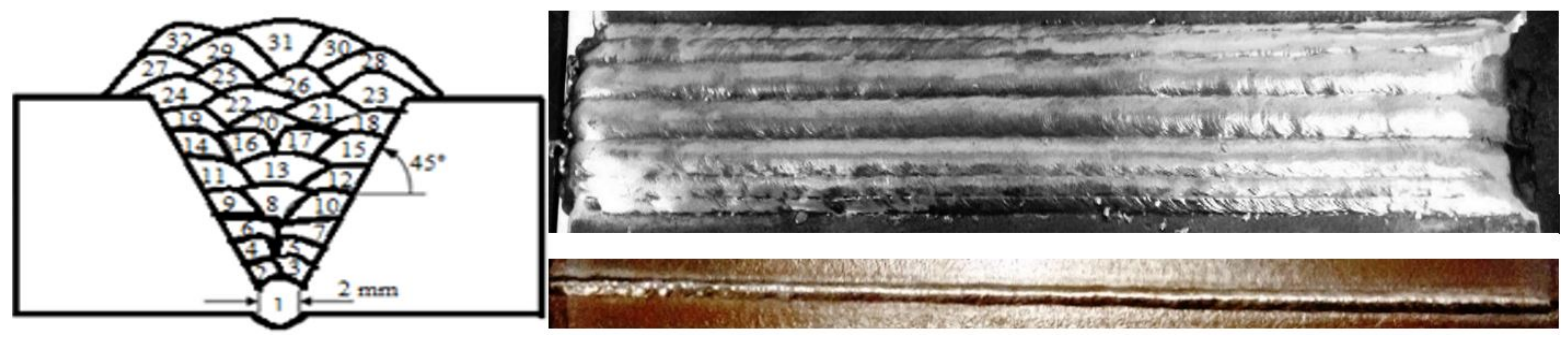

Figura 4. Sequência de deposição para a soldagem MIG-MAG. Cordão de acabamento e raiz.

A solda apresentou boa penetração com passe de raiz e enchimento coerente com o processo MIG-MAG e suficiente para análise objeto do trabalho. Após a soldagem a pesquisa segue com as caracterizações microestrutural, mecânica e analise por ressonância de barras.

\subsection{Microscopia ótica}

Para a classificação dos microconstituintes adotou-se o padrão do IIW (Instituto Internacional de Soldagem). Na Figura 5 é apresentada a microestrutura da ZF se constituindo basicamente de perlita $(P)$ e diferentes morfologias de ferrita: ferrita de contorno de grão $P F(G)$, ferrita de segunda fase alinhada FS (A) e ferrita intragranular PF (I).

Isto se deve ao fato de que as elevações da energia de soldagem decorrente dos passes subsequentes ao de raiz promoveram aumento de ferrita de contorno de grão $P F(G)$ e ferrita de segunda fase alinhada FS (A). A ferrita é a solução solida de carbono em ferro alfa (CCC) e origina-se na zona crítica, durante o resfriamento por transformação alotrópica do ferro gama (CFC). Essa é a forma estável a temperatura ambiente de alguns elementos, quando dissolvidos no ferro, tenderem a estabilizar a estrutura CCC em relação à estrutura CFC. Resultados similares de micrografia foram encontrados para Tatagiba et al. [14] e Almeida et al. [15]. Segundo estes autores, considerações devem ser feitas a influência da fração volumétrica, tamanho, morfologia, distribuição e orientação cristalografia, visto que qualquer alteração nesses quesitos aumenta os valores das constantes elásticas.

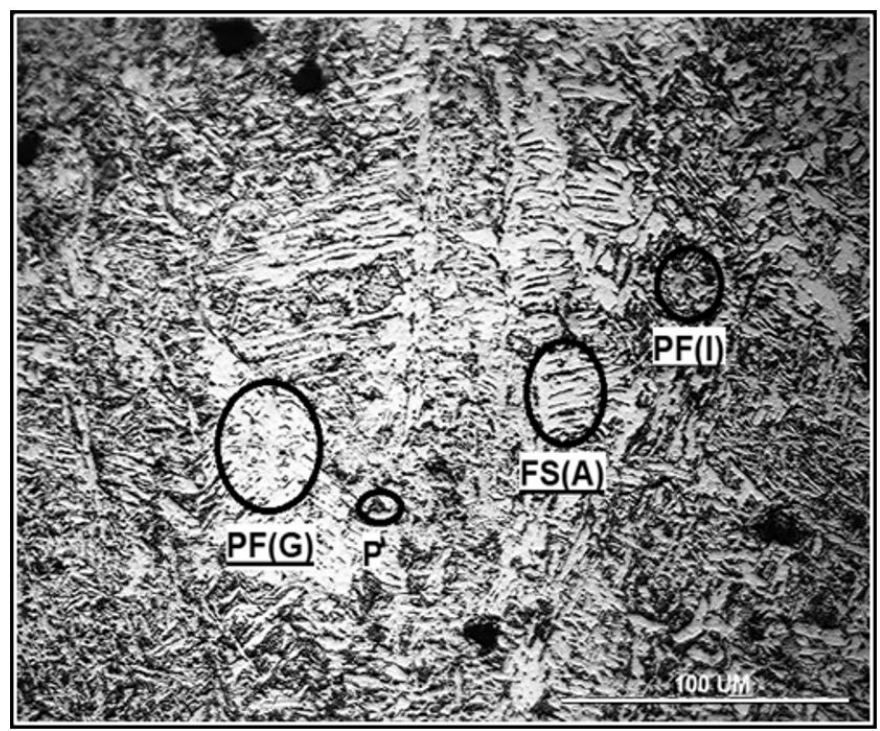

Figura 5. Micrografia da ZF do aço estrutural A36.

As quantificações volumétricas das fases presentes na junta soldada podem ser vistas na Tabela 5 ambas as fases com $\pm 3,99$ de desvio padrão.

Tabela 5. Fração volumétrica das fases.

\begin{tabular}{ccc}
\hline Amostra & Perlita (\%) & Ferrita (\%) \\
MB & 36,35 & 63,65 \\
ZF & 46,3 & 53,70 \\
\hline
\end{tabular}

Sendo: $\mathrm{MB}=$ metal base; $\mathrm{ZF}=$ zona fundida . 


\subsection{Difratrometria de raios- $X$}

A Figura 6 mostra o padrão de difração de raios-x para a ZF sobreposta ao $M B$, observa-se a manutenção das características principais dos picos do $\mathrm{MB}$ na região da ZF, logo não foi possível aferir qualquer influência provocada durante o processo de soldagem. Por este teste, no entanto, o nível de cristalinidade entre as duas regiões (MB e ZF) apresentou um alargamento na intensidade do primeiro pico para a ZF isto indica alguma alteração em nível microestrutural, como já foi observado na microscopia ótica, diferentes morfologias de ferrita e aumento volumétrico da fase pertila. Dessa maneira podem sugerir alteração nos resultados de ressonância de barras $[16,17]$.

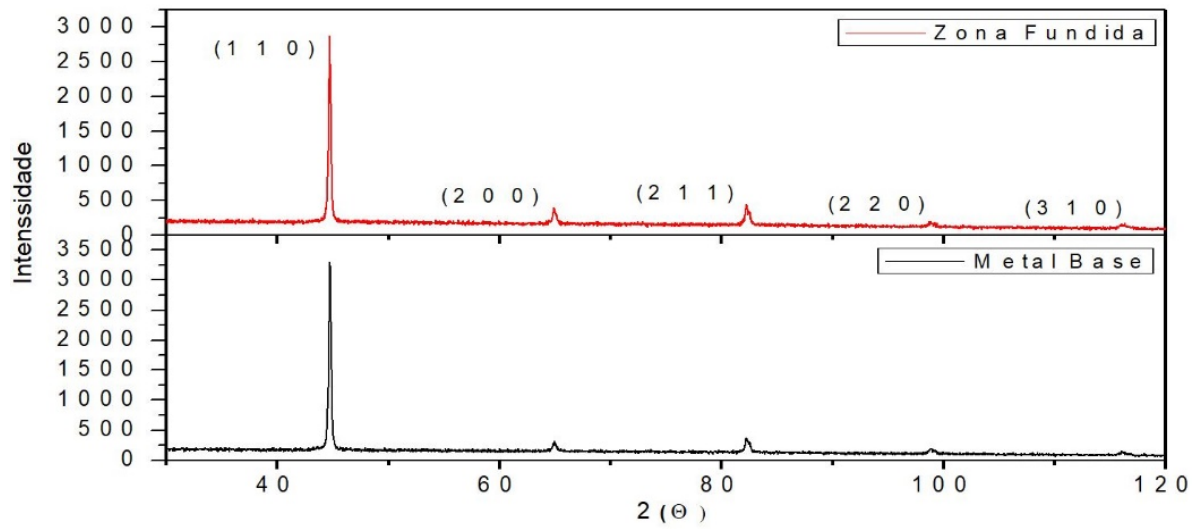

Figura 6. Difratograma de raios-x para o Metal Base e Zona Fundida.

Através da análise do difratograma, constata-se o que já se havia concluído com relação ao comportamento microestrutural do MB e da ZF. Sendo assim, não foi possível aferir o surgimento de outra fase além daquelas já constatadas nas micrografias, uma vez que não existe pico que demonstre isso.

\subsection{Tração e impacto}

O ensaio de tração comprovou a integridade das juntas soldadas em termos qualitativos e, em termos quantitativos apresentou tensão máxima de tração acima do valor mínimo de $400 \mathrm{MPa}$. A Tabela 6 mostra as propriedades adquiridas no ensaio para o MB e ZF sendo especificado a tensão de escoamento $\left(\sigma_{e}\right)$, limite de resistência a tração (LR) e alongamento $(\Delta \mathrm{l})$.

Tabela 6. Propriedade mecânica obtida no ensaio de tração.

\begin{tabular}{|c|c|c|c|c|c|}
\hline \multirow{2}{*}{ Propriedade } & \multicolumn{4}{|c|}{ Material } & \multirow{2}{*}{ Norma [4] } \\
\hline & MB1 & MB2 & ZF1 & ZF2 & \\
\hline (LR) (MPa) & 536,63 & 507,60 & 522,68 & 511,8 & $400-550$ \\
\hline$\left(\sigma_{e}\right)(\mathrm{MPa})$ & 502,87 & 477,56 & 451 & 458 & 250 \\
\hline$(\Delta \mathrm{L})$ & $32,92 \%$ & $33,33 \%$ & $34,05 \%$ & $40,2 \%$ & 20 \\
\hline
\end{tabular}

Sendo: $\mathrm{MB}=$ Metal base; $\mathrm{ZF}=$ Zona fundida.

Os resultados do ensaio de resistência ao impacto para as duas regiões são apresentados na Tabela 7, foi verificado uma tenacidade média menor para a região com entalhe na ZF.

Tabela 7. Energia absorvida no Metal Base e Zona Fundida.

\begin{tabular}{|c|c|c|c|c|}
\hline Corpo de prova & Posição do entalhe & Energia absorvida (J) & Média & Norma [4] \\
\hline 1 & & 58,5 & & \multirow{6}{*}{$35 \mathrm{~J}$} \\
\hline 2 & $\mathrm{ZF}$ & 56,5 & $57,33 \pm 0,85$ & \\
\hline 3 & & 57 & & \\
\hline 1 & & 72,5 & & \\
\hline 2 & $\mathrm{MB}$ & 67,5 & $68,67 \pm 2,77$ & \\
\hline 3 & & 66 & & \\
\hline
\end{tabular}

Sendo: $\mathrm{ZF}$ = Zona fundida; $\mathrm{MB}=$ Metal base. 


\subsection{MEV e EDS da fratura}

As superfícies de fratura de ambos os metais de solda dos corpos de prova de tração e charpy apresentaram fratura dúctil de coalescência de microvazios (CMV). Resultado similar foi observado no trabalho de Kaçar e Kökemli [18], concordando com Sajid e Kiran [19] onde a maior zona de fratura por CMV reflete a maiores resultados de ductilidade e alta absorção de energia de impacto algo que se verifica neste trabalho com médias de impacto acima da especificada em norma no ensaio Charpy. A região da fratura foi analisada por espectrometria de energia dispersiva de raios-x (EDS) acoplado ao MEV, as Figuras 7a fratura de tração com espectro de EDS e Figura 7b fratura por impacto com espectro de EDS, mostram, com aumento 500x, imagem do MEV dessas regiões analisada.
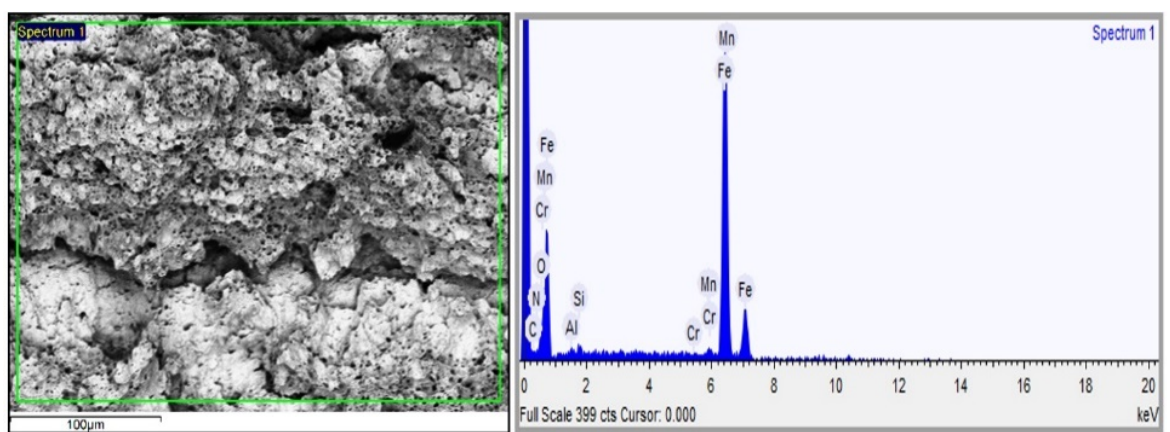

(a)
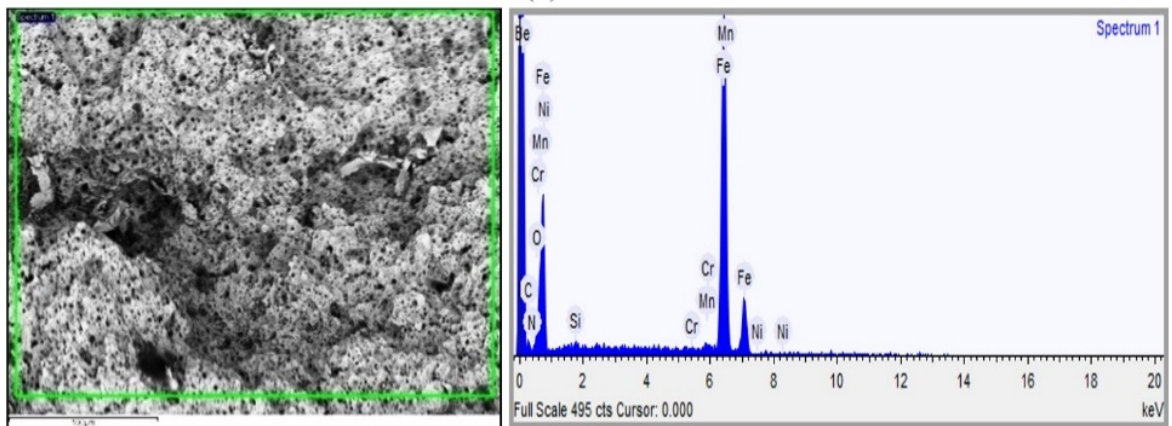

(b)

Figura 7. Imagem MEV (a) Tração; (b) Charpy V.

Após análise realizada por EDS, revelou-se a composição química da fratura da ZF das amostras ensaiadas por tração e impacto, como mostrado na Tabela 8.

Tabela 8. Resultado do EDS da fratura da ZF dos ensaios de impacto e tração.

\begin{tabular}{ccccccccc}
\hline & \multicolumn{7}{c}{ Elemento \% (Peso) } \\
\cline { 2 - 9 } Ensaio (ZF) & $\mathbf{C}$ & $\mathbf{N i}$ & $\mathbf{0}$ & $\mathbf{A l}$ & $\mathbf{S i}$ & $\mathbf{C r}$ & $\mathbf{M n}$ \\
Tração & 0,1832 & 1,263 & 1,285 & 0,393 & 0,553 & 0,16 & 0,949 \\
Impacto & 0,1531 & 2,015 & 0,958 & - & 0,329 & 0,124 & 0,769 \\
\hline
\end{tabular}

Sendo: ZF = Zona fundida.

A composição média, em \% peso, nas regiões de fratura foi de 0,168\% C; 1,64\% Ni; 1,1215\% 0; 0,441\% Si; 0,142\% Cr; 0,859 Mn. O maior teor de carbono observado por EDS explica a causa da menor tenacidade observada no ensaio de impacto e a explicação da maior dureza dessa região. Svensson e Gretoft [20] observaram comportamento contrário na região da ZF, para o metal de solda com menor teor de carbono foi observado, inesperadamente, baixa tenacidade, o autor atribuiu esse comportamento ao conteúdo de nitrogênio.

Segundo Zhang e Farrar [21] o Mn e o Ni têm função similar, influenciam na formação da ferrita acicular (AF) e ferrita de contorno de grão $P F(G)$, aumentando a dureza. Além disso, o teor de níquel refina o tamanho do grão e baixa a tenacidade dessa região. Para Jorge et al. [22], o cromo prejudica a resistência ao impacto, embora promova um aumento na porcentagem de (AF). No estudo do autor, um aumento de carbono promoveu uma diminuição da energia absorvida, onde atribuiu esse comportamento á maior fração volumétrica de martensita-austenita. 


\subsection{Microdureza}

A Figura 8a mostra o diagrama de distribuição de endentações. Na primeira linha de reforço e raiz a microdureza Vickers média nessa região obtida foi de $206 \mathrm{HV}$ com desvio padrão de $\pm 12,9$. O comportamento do material para a região da junta soldada é observado uma média dessa região e está na faixa de resultados aceitáveis para o aço A36.

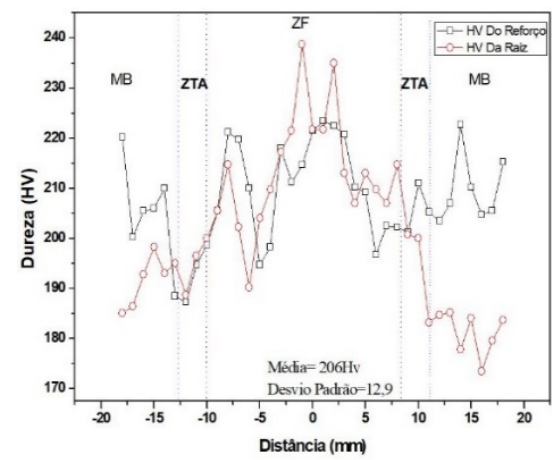

(a)

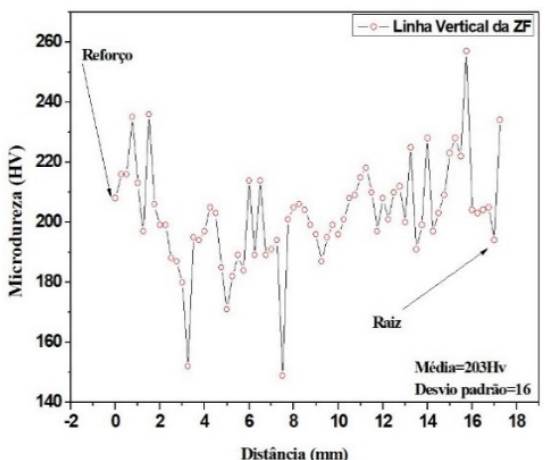

(b)

Figura 8. (a) Distribuição de microdureza no passe de acabamento e na raiz; (b) Microdureza na Zona Fundida. Hv = Microdureza Vickers; $\mathrm{MB}=$ Metal base; $\mathrm{ZTA}=$ Zona termicamente afetada pelo calor; $\mathrm{ZF}=$ Zona fundida.

O comportamento da microdureza na zona termicamente afetada para ambas as amostras apresenta um crescente aumento à medida que se aproxima da ZF, esse comportamento foi verificado no trabalho de Abson [23]. A maior microdureza registrada na ZF nos remete a valores de tenacidade menores nessa região resultado similar aos trabalhos de Kaçar e Kökemli [18] e Tatagiba et al. [14]. A microdureza da região da ZF foi quantificada na direção vertical, sentido topo (acabamento) para baixo (raiz) no centro da ZF com uma carga de 4950N (0,5HV). A Figura 8b exibe o resultado de microdureza na ZF.

O comportamento do material da ZF exibe uma microdureza Vickers média de $203 \mathrm{HV}$ com desvio padrão de \pm 16 , próxima dos valores aceitáveis para o $\mathrm{MB}$ que neste estudo ficou com média de $180 \mathrm{HV}$ sendo aceito na literatura valores de até $200 \mathrm{HV}$. A dureza na região da raiz apresenta um pico com o maior patamar registrado, isso pode ser atribuído a maior diluição nessa região ou descontinuidades segundo observou Trindade [24] em seu trabalho. Neste trabalho considera-se a influência da diluição, uma vez que não foram constatadas descontinuidades na junta soldada.

\subsection{Caracterização elástica}

\subsubsection{Quantificação dos valores dos módulos elásticos em função da distância dos apoios para o MB}

A quantificação do módulo de Young, cisalhamento e coeficiente de Poisson em função da distância do acoplamento dos transdutores emissor e receptor um conjunto de medidas foi realizado com o MB e está apresentado na Tabela 9, já foi mencionado que a distância de $48,85 \mathrm{~mm}$ é a distância nominal para um corpo de prova com $88,5 \mathrm{~mm}$ de comprimento.

Tabela 9. Quantificação das constantes elásticas em função da distância entre os apoios.

\begin{tabular}{|c|c|c|c|c|c|c|}
\hline $\begin{array}{l}\text { Distância } \\
\text { entre os } \\
\text { apoios }\end{array}$ & $\begin{array}{l}\text { Variação em relação à } \\
\text { variação nominal (mm) }\end{array}$ & $\begin{array}{l}\text { Frequência de } \\
\text { ressonância } \\
\text { flexional } \\
\text { (KHz) }\end{array}$ & $\begin{array}{l}\text { Frequência de } \\
\text { ressonância } \\
\text { torcional } \\
(\mathrm{KHz})\end{array}$ & $\begin{array}{l}\text { Módulo de } \\
\text { Young (GPa) }\end{array}$ & $\begin{array}{c}\text { Módulo de } \\
\text { cisalhamento } \\
\text { (GPa) }\end{array}$ & $\begin{array}{l}\text { Coeficiente de } \\
\text { Poisson }\end{array}$ \\
\hline 44,07 & -5 & 4272,6 & 12252,2 & 199,36 & 77,87 & 0,28 \\
\hline 45,07 & -4 & 4444,5 & 12148,6 & 216 & 76,56 & 0,41 \\
\hline 46,07 & -3 & 4333,3 & 12148,6 & 205,36 & 76,56 & 0,34 \\
\hline 47,07 & -2 & 4444,5 & 12185,6 & 216,29 & 77,03 & 0,40 \\
\hline 48,07 & -1 & 4222,2 & 12037,5 & 194,74 & 75,17 & 0,30 \\
\hline 49,07 & 0 & 4333,3 & 12148,6 & 205,32 & 76,56 & 0,34 \\
\hline 50,07 & 1 & 4222,2 & 12074,5 & 205,39 & 75,63 & 0,36 \\
\hline 51,07 & 2 & 4222,2 & 12074,5 & 205,39 & 75,63 & 0,36 \\
\hline 52,07 & 3 & 4222,2 & 12111,6 & 194,68 & 76,1 & 0,28 \\
\hline 53,07 & 4 & 4333,4 & 12148,6 & 194,66 & 76,56 & 0,27 \\
\hline 54,07 & 5 & 4333,3 & 12111,6 & 194,68 & 76,1 & 0,28 \\
\hline
\end{tabular}


A Tabela 10 apresenta os efeitos dos resultados para a quantificação dos módulos de elasticidade e frequências fundamentais em função da variação das distancias entre o acoplamento.

Tabela 10. Efeito do deslocamento sobre as constantes elásticas e frequências fundamentais de flexão e torção.

\begin{tabular}{lcc}
\hline \multicolumn{1}{c}{ Efeito } & Deslocamento positivo & Deslocamento negativo \\
Frequência Fundamental Flexional (KHz) & $-1,54 \%$ & $+0,23 \%$ \\
Frequência Fundamental Torcional (KHz) & $-0,37 \%$ & $+0,049 \%$ \\
Módulo de Young (GPa) & $-3,1 \%$ & $+0,50 \%$ \\
Módulo de cisalhamento (GPa) & $-0,007 \%$ & $+0,10 \%$ \\
Razão de Poisson & $-8,82 \%$ & $+1,76 \%$ \\
\hline
\end{tabular}

Para o MB, em termos estatísticos, o módulo dinâmico de Young o seu deslocamento negativo nada influenciou, pois, o mesmo está na faixa do desvio padrão do teste, já o deslocamento positivo o módulo diminuiria em 1,1\% que significa quantificar em termos de $0,454 \mathrm{GPa} / \mathrm{mm}$. O módulo de cisalhamento no deslocamento negativo aumenta em $0,05 \%$ ou $0,0079 \mathrm{GPa} / \mathrm{mm}$ e no deslocamento positivo nada influenciou. O coeficiente de Poisson no deslocamento negativo nada influenciou e no deslocamento positivo ocorreu $2,26 \%$ ou $0,0053 / \mathrm{mm}$. Suterio [10] observou resultado similar onde $1 \mathrm{~mm}$ pouco influência nas medidas das constantes elásticas.

\subsubsection{Ressonância de barras modo flexional e torcional}

Após a soldagem o material da ZF utilizado para o trabalho foi usinado por um processo de corte e retifica. As dimensões das amostras em milímetros foram medidas com um paquímetro com duas casas decimais. A caracterização elástica feita na ZF foi feita com dimensões iguais ao corpo de prova do MB, no entanto uma diferença de $2,427 \mathrm{~g}$ de massa foi registrada, a Figura 9 mostra os espectros de ressonância para os três primeiros picos de flexão e torção, ensaios realizados no modo Kaneko e ASTM 1876 apresentaram resultados semelhantes, pois os picos fundamentais de flexão e torção foram encontrados com exatidão no primeiro modo de frequência fundamental. Observa-se uma intensidade em todos os picos fundamentais de ressonância na ZF em relação ao $\mathrm{MB}$, tal comportamento é um indicativo do resultado de soldagem dos vários passes como aumento da composição química e efeito dos ciclos térmicos e reaquecimento experimentados nessa região.

A verificação de precisão dos picos para os corpos de prova de MB e ZF pode ser observada na Tabela 11, comparando-se a razão entre picos fundamentais para o modo flexional e torcional. Os resultados mostram que o maior erro percentual na ZF entre os resultados experimentais e teóricos foi de 2,23\% para frequência flexional e 0,20\% para frequência torcional.

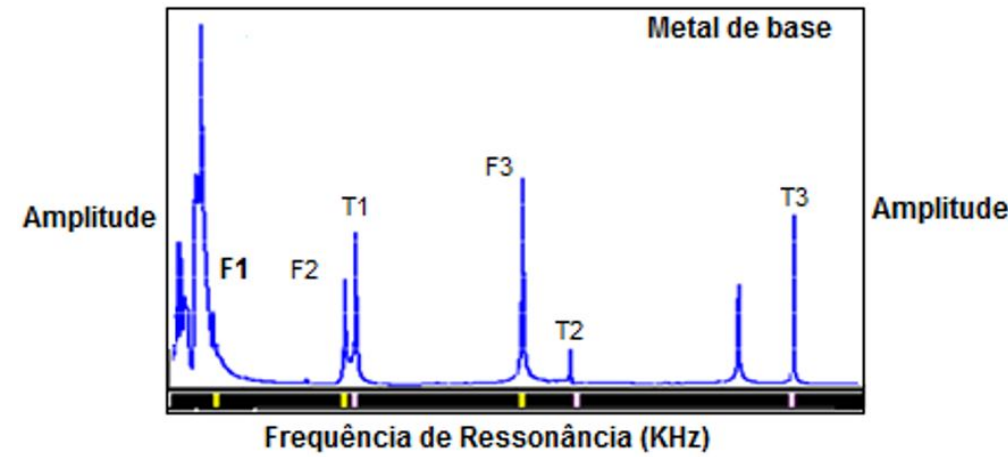

(a)

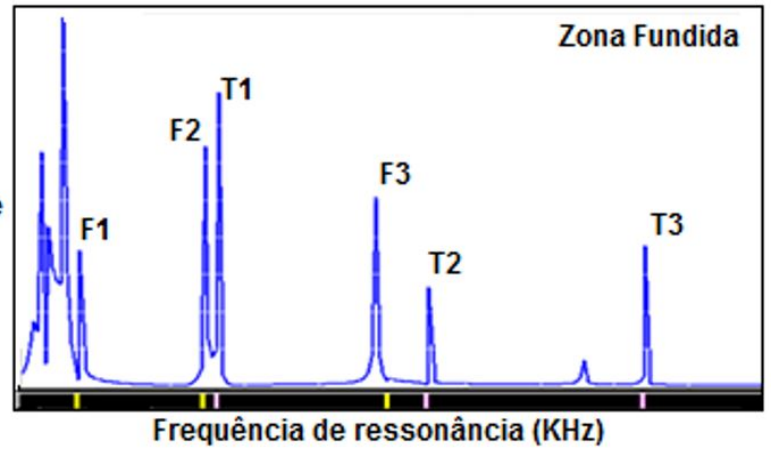

(b)

Figura 9. Espectro de varredura de frequências: (a) Para o metal base; (b) Para a zona fundida. F1, F2 e F3 são: Primeiro, segundo e terceiro picos de flexão; T1,T2 e T3 são: Primeiro, segundo e terceiro picos de torção respectivamente.

A influência das diferenças constatadas na Tabela 11 para os módulos dinâmicos de Young, cisalhamento e Poisson pode ser vista na Tabela 12. 
Tabela 11. Erro padrão de picos de flexão e torção para MB e ZF.

\begin{tabular}{|c|c|c|c|c|c|}
\hline \multirow{2}{*}{ Razão entre as frequências } & \multirow{2}{*}{ Previsão teórica } & \multicolumn{2}{|c|}{ Resultado experimental } & \multicolumn{2}{|c|}{ Erro padrão (\%) } \\
\hline & & MB & ZF & MB & ZF \\
\hline $\mathrm{F} 2 / \mathrm{F} 1$ & 2,6431 & 2,707 & 2,702 & 2,42 & 2,23 \\
\hline F3/F1 & 4,9952 & 5,01 & 5,026 & 0,30 & 0,62 \\
\hline $\mathrm{T} 2 / \mathrm{T} 1$ & 2 & 2,01 & 1,996 & 0,50 & 0,20 \\
\hline T3/T1 & 3 & 2,94 & 3,004 & 2 & 0,13 \\
\hline
\end{tabular}

Sendo: MB = Metal base; ZF = Zona fundida; F1,F2 e F3 são: Primeiro, segundo e terceiro picos de flexão; T1,T2 e T3 são: Primeiro, segundo e terceiro picos de torção respectivamente.

Tabela 12. Módulos elásticos medidos no MB e ZF para mesma geometria.

\begin{tabular}{|c|c|c|c|c|c|}
\hline Amostra & E (GPa) & G (GPa) & $\mu$ & Ff (Hz) & Ft $(\mathrm{Hz})$ \\
\hline $\mathrm{MB}$ & $206,08 \pm 4,53$ & $79,775 \pm 0,05$ & $0,30 \pm 0,02$ & $4291,4 \pm 39,18$ & $12120,5 \pm 3,4$ \\
\hline ZF & $216,17 \pm 3,33$ & $85,48 \pm 0,4$ & $0,26 \pm 0,01$ & $4399,6 \pm 33,09$ & $12674 \pm 29,68$ \\
\hline
\end{tabular}

Sendo: $\mathrm{MB}=$ Metal base; $\mathrm{ZF}=$ Zona fundida.

Uma média ligeiramente maior encontrada para os módulos dinâmicos na região da ZF: 4,9\% maior para o módulo dinâmico de Young, 7,65\% maior para o cisalhamento e 11,7\% menor para a razão de Poisson, devido possivelmente as fases presentes, era esperado, no entanto a discussão é complexa uma vez que a microestrutura do material tem uma combinação de dois materiais distintos com uma morfologia similar, porém mais refinado na zona fundida onde se verificou uma alteração química de elementos que tem uma função metalúrgica especifica.

A diminuição do tamanho do grão é um indicativo de aumento de módulos dinâmicos, tal relação foi constatada por Latella e Humphries [25] e Montecinos et al. [26] utilizando a técnica de excitação por impulso.

$\mathrm{O} \mathrm{MB}$ apresentou fração volumétrica menor de perlita em comparação a ZF e a dureza média foi $10 \%$ menor que a da zona fundida. $\mathrm{O}$ modo de ruptura dos materiais constatado por MEV, com maior índice de fratura transgranular, para o MB pode ser um indicativo do modo de propagação das ondas no material entre grãos. Tanaka et al. [16] concluiu que o movimento atômico ao longo dos contornos de grão e limites de grão causa a rápida diminuição do módulo de Young.

A determinação de propriedades mecânicas na região plástica pelo método dinâmico, por exemplo, a determinação do escoamento, é complexa e necessita de mais estudo, Paiva [2] menciona que métodos dinâmicos são muito rápidos e assim, deformações plásticas dependentes do tempo podem não ser medidas. Por outro lado, a medida pelo método estático é comparativamente mais longa e os resultados podem ser afetados por esta deformação plástica.

\subsubsection{Teste de homogeneidade e isotropia}

A homogeneidade do material foi verifica na ZF a metodologia seguiu a mesma metodologia realizada para o MB. As Figuras 10a e 10b mostram respectivamente a relação entre a frequência de ressonância com os módulos de Young e modulo de cisalhamento para a região da ZF o ensaio foi realizado em direções diferentes e os resultados analisados por regressão linear das medidas realizadas. O grau de homogeneidade da ZF foi de 0,9972 para o modo flexional e de 0, 99959 para o modo torcional. Autores como Blessing [27] e Chanbi et al. [10] obtiveram a partir da técnica dinâmica comprovações da homogeneidade dos materiais. A homogeneidade apresentada na ZF teve índice maior em comparação ao MB isso é atribuído a microestrutura da ZF ser de morfologia diferente e em fração volumétrica diferente e ainda pela composição química.

O maior teor de carbono observado pelo EDS resultou em uma dureza maior na ZF, logo maior resistência dessa região. Em nível atômico as constantes elásticas são susceptíveis a mudanças quando exposto a mudanças térmicas, algo que pode ser explicado para a ZF que teve áreas reaquecidas no processo de soldagem, logo as tensões residuais [1,2] são um indicativo da maior variação observada para as frequências ressonantes na ZF. A Tabela 13 resume as principais relações feitas no estudo. 


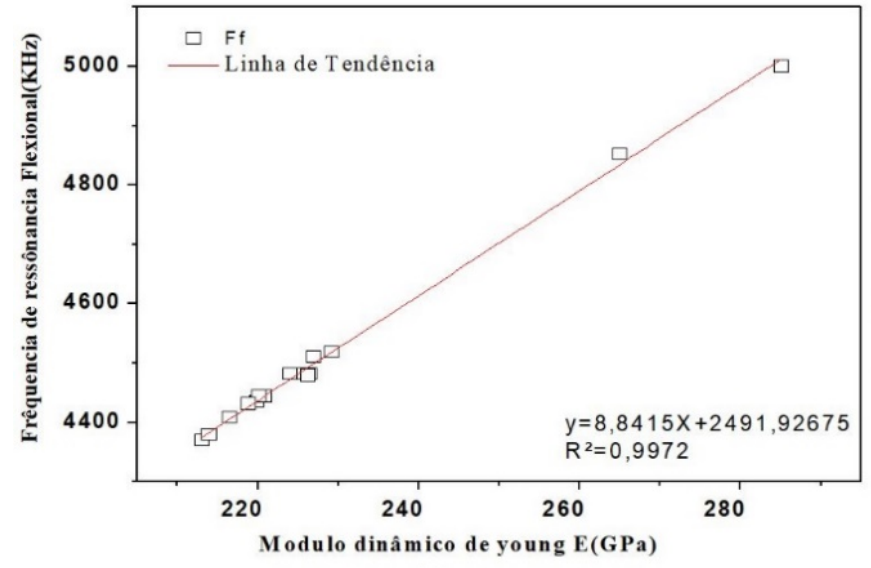

(a)

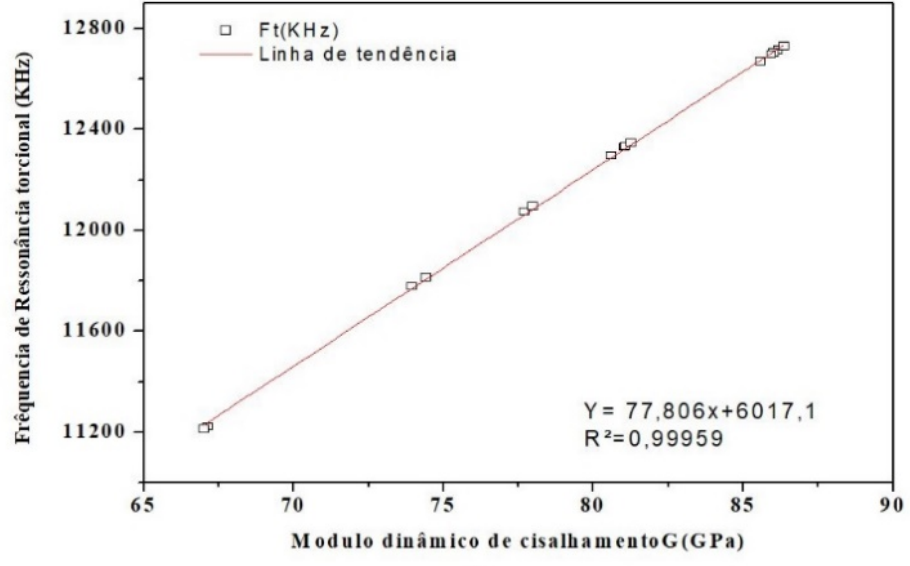

(b)

Figura 10. Linha de tendência: (a) Frequência $\times$ Módulo dinâmico de Young; (b) Frequência $\times$ Cisalhamento. Ff = Frequência de ressonância flexional; Ft= Frequência de ressonância torcional.

Tabela 13. Resumo das principais relações.

\begin{tabular}{|c|c|c|c|}
\hline Propriedade & MB & ZF & Relação na ZF \\
\hline Elemento químico & Aço A36 & Adição de elementos & Aumentou \\
\hline \multicolumn{4}{|c|}{ Caracterização Microestrutural } \\
\hline \multirow{2}{*}{ Fração volumétrica } & $P=36,45$ & $P=46,29$ & Perlita (Aumentou) \\
\hline & $F=63,65$ & $P=53,703$ & Ferrita (Diminuiu) \\
\hline \multicolumn{4}{|c|}{ Caracterização Mecânica } \\
\hline Dureza (HV) & 180 & 203 & Aumentou \\
\hline Tenacidade (J) & $68,67 \pm 2,77$ & $57,33 \pm 0,85$ & Diminuiu \\
\hline$\sigma_{\mathrm{e}}(\mathrm{MPa})$ & $490,2 \pm 12,6$ & $454,5 \pm 3,5$ & Diminuiu \\
\hline $\mathrm{LR}(\mathrm{MPa})$ & $562,115 \pm 14,5$ & $557,24 \pm 5,4$ & Diminuiu \\
\hline$\Delta \mathrm{l}$ & 33,125 & 37,125 & Aumentou \\
\hline \multicolumn{4}{|c|}{ Caracterização Elástica } \\
\hline $\mathrm{E}(\mathrm{GPa})$ & $206,08 \pm 4,53$ & $216,17 \pm 3,33$ & Aumentou \\
\hline $\mathrm{G}(\mathrm{GPa})$ & $79,37 \pm 0,05$ & $85,48 \pm 0,4$ & Aumentou \\
\hline Poisson & $0,30 \pm 0,02$ & $0,265 \pm 0,01$ & Diminuiu \\
\hline$F_{f}(K H z)$ & $4291,4 \pm 39,18$ & $4399,6 \pm 33,09$ & Aumentou \\
\hline $\mathrm{F}_{\mathrm{t}}(\mathrm{KHz})$ & $12120,5 \pm 3,4$ & $12674 \pm 29,7$ & Aumentou \\
\hline Massa (g) & 65 & 67,427 & Aumentou \\
\hline $\mathrm{R}^{2}$ & 0,99 & 0,99 & - \\
\hline
\end{tabular}

Sendo: $\mathrm{MB}=$ Metal base; $\mathrm{ZF}=$ Zona fundida.

A partir da Tabela 13, observa-se um acréscimo de elementos químico o que era esperado, além de um aumento do teor de perlita e uma diminuição das propriedades mecânicas da região da zona fundida em relação ao metal base isso se deve a menor espessura do material da ZF que foi submetido a ensaio de tração. Comportamento similar foi observado por Sajid e Kiran [19] e Trindade et al. [24].

O método aplicado na zona fundida foi eficiente, apresentando resultados mais elevados em relação ao MB, mas dentro de uma faixa esperada. Este resultado é justificado pelo maior teor de carbono observado pelo EDS que resultou em uma dureza maior na ZF, logo, maior resistência dessa região, menor tenacidade e maior fração volumétrica de fase perlita com diferentes morfologias de ferrita devido ao processo de soldagem.

É comum a diminuição das constantes elásticas quando se tem um aumento do tamanho do grão, algo que aconteceu ao inverso neste trabalho, com o refinamento do tamanho do grão para a ZF onde as constantes aumentaram apesar de terem um coeficiente de determinação médio $\mathrm{R}^{2}$ de $0,99 \%$ o que indica que o fator elemento químico e microestrutural são determinantes. 


\section{Conclusões}

Sobre a caracterização elástica de juntas soldadas pela técnica de ressonância de barras os resultados e discussão permitem concluir que:

- A resistência a tração, limite de escoamento e alongamento da ZF ficaram dentro dos padrões estabelecidos por norma. A aplicação da técnica de microscopia ótica, DRX, MEV e EDS da ZF demonstraram a formação de fase típica sem a formação de novas fases, o comportamento de fratura se mostrou dúctil. O refino de grão na região da ZF revelou uma maior quantidade de perlita e maior teor de elementos de liga;

- $\quad$ O método de medida das propriedades mecânicas por ressonância de barras é viável tanto para prever no MB e na ZF os valores das constantes elásticas, bem como se mostrou eficiente em prever a homogeneidade do material. Os coeficientes das regressões foram superiores a 99\%, essa correlação é considerada altamente significativa, no entanto não foi possível se correlacionar com as propriedades no regime plástico;

- $\quad$ O método de ressonância de barras no modo flexional e torcional revelam-se importante ferramenta para inferência não-destrutiva dos módulos de elasticidade da ZF, os quais sofreram uma pequena influência na medida das constantes elásticas em relação ao MB de 4,9\% a mais para o módulo de Young, 7,65\% maior para o cisalhamento e 11,7\% menor para a razão de Poisson;

- As diferenças dos resultados para as constantes elásticas do MB e ZF são atribuídas à densidade do material, a composição química e a microestrutura. A composição química, massa e microestrutura verificada na ZF são um indicativo da diferença constatada nos ensaios de ressonância tanto para caracterização das constantes elástica como para o teste de homogeneidade.

\section{Agradecimentos}

A FAPEMA e ao IFMA, Campus Monte Castelo pelo apoio recebido.

\section{Referências}

[1] American Society for Testing and Materials. ASTM E1875-13: standard test method for dynamic Young's modulus, shear modulus, and Poisson's ratio by sonic resonance. West Conshohocken: ASTM; 2013. http://dx.doi.org/10.1520/E1875-13.

[2] Paiva AEM. Desenvolvimento de um equipamento para avaliar propriedades elásticas de cerâmicas refratárias a altas temperaturas pelo método de ressonância de barras [tese de doutorado]. São Carlos: Universidade Federal de São Carlos; 2002.

[3] Pereira AHA, Venet M, Tonnesen T, Rodrigues JA. Desenvolvimento de um equipamento para a caracterização não-destrutiva dos módulos elásticos de materiais. Cerâmica. 2010;56(338):118-122. http://dx.doi.org/10.1590/\$0366-69132010000200004.

[4] American Society for Testing and Materials. ASTM A36/36M: standard specification for carbon structural steel: annual book of ASTM standards. West Conshohocken: ASTM; 2008. 4 p.

[5] American Welding Society. AWS A 5.18: specification for carbon steel electrodes and rods for gas shielded arc welding. Miami: AWS; 2005.

[6] American Society for Testing and Materials. ASTM E384-17: standard test method for micro indentation hardness of materials. West Conshohocken: ASTM; 2017. http://dx.doi.org/10.1520/E0384-17.

[7] American Society for Testing and Materials. ASTM E23-18: standard test methods for notched bar impact testing of metallic materials. West Conshohocken: ASTM; 2018. http://dx.doi.org/10.1520/E0023-18.

[8] Tewary NK, Kundu A, Nandi R, Saha JK, Ghosh SK. Microstructural characterization and corrosion performance of old railway girder bridge steel and modern weathering structural steel. Corrosion Science. 2016;113:57-63. http://dx.doi.org/10.1016/j.corsci.2016.10.004.

[9] Guzman-Flores I, Vargas-Arista B, Gasca-Dominguez JJ, Cruz-Gonzalez CE, González-Albarrán MA, del Prado-Villasana J. Effect of torch weaving on the microstructure, tensile and impact resistances, and fracture of the HAZ and weld bead by robotic GMAW process on ASTM A36 steel. Soldagem e Inspeção. 2017;22(1):72-86. http://dx.doi.org/10.1590/0104-9224/si2201.08.

[10] Suterio R. Caracterização de constantes elásticas de materiais isotrópicos por holografia óptica eletrônica combinada ao método de ressonância mecânica [dissertação de mestrado]. Florianópolis: Universidade Federal de Santa Catarina; 1998.

[11] Chanbi D, Ogam E, Amara SE, Fellah ZEA. Synthesis and mechanical characterization of binary and ternary intermetallic alloys based on Fe-Ti-Al by resonant ultrasound vibrational methods. Materials. 2018;11(5):746. http://dx.doi.org/10.3390/ma11050746. PMid:29735946.

[12] Bao Y, Zhang H, Ahmadi M, Karim MA, Wu HF. Measurements of Young's and shear moduli of rail steel at elevated temperatures. Ultrasonics. 2014;54(3):867-873. http://dx.doi.org/10.1016/j.ultras.2013.10.015. PMid:24268178. 
[13] Likhachev DV. Evaluation of different dispersion models for correlation of spectroscopic ellipsometry and X-ray reflectometry. The Review of Scientific Instruments. 2019;90(2):023909. http://dx.doi.org/10.1063/1.5050492. PMid:30831715.

[14] Tatagiba LCS, Silva AGP, Paranhos R. Caracterização de uma junta soldada com suporte de solda a base de fibra de vidro. Soldagem e Inspeção. 2012;17(1):11-18. http://dx.doi.org/10.1590/S0104-92242012000100003.

[15] Almeida LLP, Tatagiba LCS, Rosenthal R, Paranhos RPR. Soldagem unilateral com suporte cerâmico de cordierita. Soldagem e Inspeção. 2010;15(1):61-70. http://dx.doi.org/10.1590/S0104-92242010000100008.

[16] Tanaka H, Qun LF, Munekata O, Taguchi T, Narita T. Elastic properties of Sn-based Pb-free solder alloys determined by ultrasonic pulse echo method. Materials Transactions. 2005;46(6):1271-1273. http://dx.doi.org/10.2320/matertrans.46.1271.

[17] Tognana S, Salgueiro W, Somoza A, Marzocca A. Measurement of the Young's modulus in particulate epoxy composites using the impulse excitation technique. Materials Science and Engineering A. 2010;527(18-19):4619-4623. http://dx.doi.org/10.1016/j.msea.2010.04.083.

[18] Kaçar R, Kökemli K. Effect of controlled atmosphere on the mig-mag arc weldment properties. Materials \& Design. 2005;26(6):508-516. http://dx.doi.org/10.1016/j.matdes.2004.07.027.

[19] Sajid HU, Kiran R. Influence of stress concentration and cooling methods on post-fire mechanical behavior of ASTM A36 steels. Construction \& Building Materials. 2018;186:920-945. http://dx.doi.org/10.1016/j.conbuildmat.2018.08.006.

[20] Svensson LE, Gretoft B. Microstructure and impact toughness of C-Mn weld metals. Welding Journal. 1990;69(12):454.

[21] Zhang Z, Farrar RA. Influence of $\mathrm{Mn}$ and $\mathrm{Ni}$ on the microstructure and toughness of C-Mn-Ni weld metals. Welding Journal. 1997;76(5):183.

[22] Jorge JCF, Souza LFG, Rebello JMA. The effect of chromium on the microstructure / toughness relationship of C-Mn weld metal deposits. Materials Characterization. 2001;47(3-4):195-205. http://dx.doi.org/10.1016/S1044-5803(01)00168-1.

[23] Abson DJ. Microstructure and mechanical properties of gas-shielded welding of C-Mn-Ni steel. Welding Journal. 1993;72(5):173-187.

[24] Trindade VB, Alves SMS, Cândido LC, Faria GL, Porcaro RR. Características microestruturais e mecânicas ao longo da seção transversal de juntas soldadas em multipasses pelo processo GMAW de um aço API 5L X65Q. Soldagem e Inspeção. 2017;22(2):217-227. http://dx.doi.org/10.1590/0104-9224/si2202.10.

[25] Latella BA, Humphries SR. Young's modulus of 2.25 Cr-1Mo steel at elevated temperature. Scripta Materialia. 2004;51(7):635-639. http://dx.doi.org/10.1016/j.scriptamat.2004.06.028.

[26] Montecinos S, Tognana S, Salgueiro W. Determination of the Young's modulus in CuAlBe shape memory alloys with different microstructures by impulse excitation technique. Materials Science and Engineering A. 2016;676:121-127. http://dx.doi.org/10.1016/j.msea.2016.08.100.

[27] Blessing G. The pulsed ultrasonic velocity method for determining material dynamic elastic moduli. In: Wolfenden A. STP1045-EB: dynamic elastic modulus measurements in materials. West Conshohocken: ASTM International; 1990. p. 47-57. http://dx.doi.org/10.1520/STP24614S. 